\title{
Deer numbers
}

How many deer? A field guide to estimating deer population size. B. A. Mayle, A. J. Peace and R. M. A. Gill. The Forestry Commission, Edinburgh, 1999, 96 pp., $£ 14.00$ (pbk). ISBN 0855384050 .

Deer are among most successful groups of animals. During few recent decades they extended their range and increased their numbers in most countries of Europe and North America. When numerous, they become noxious. They inflict damage to forest regeneration by browsing seedlings and debarking saplings. They also feed heavily on farm crops, causing troublesome assessments of the extent of damage and payments of compensations. Their high reproductive potential makes it that their population easily get out of control.

This fact brings about the need of precise management. And precise management is possible only on the basis of a reliable estimate of numbers of an object of management.

This booklet is on techniques and procedures of the estimation of deer numbers. In five chapters it presents a neatly arranged review of existing methods. The first, very short chapter contains the rationale why we should count deer. It appears that we do this in order to identify the population trend and to help in determining the extent of sustainable cull. The knowledge of the status of population will also assist in the reduction of population size. Practical reasons of reducing population size are: alleviation of damage in farm and forest crops, decrease in accidents on roads, and the improvement of herd quality.

The second chapter advises reader how to select a proper method to achieve his goals. First of all the objective (s) have to be clearly and precisely stated. Only then an appropriate and efficient method can be selected. Authors dwell on such characteristics of sampling procedure, as accuracy, precision and bias. They describe ways of calculation of the maximum sample size under constrains of a fixed budget.

The third chapter presents no less than 21 methods of population estimation. These are described according to an uniform format including such important points as kind of data records, needed equipment, advantages and disadvantages of the technique, etc. Descriptions are supported by worked examples in most cases. This chapter forms the main body of the booklet. Methods presented are grouped as: direct counts (5 methods), night counts (3 methods), other direct methods (2), indirect methods (2), faecal pellet counts (5), and population reconstructions from mortality data (4 methods).

Very brief chapter 4 summarizes the importance of accurate population estimates which will be required since deer populations will likely continue to increase their size and extend distribution range in years to come.

The fifth chapter is rather a conglomerate of useful appendices, as random number table, various forms, data on the rate of dung decay, etc.

List of references includes only the essential publications used by the authors.

Careful reading of book revealed very few inconsistencies and a slight bias towards the situation prevailing on British Isles which after all differs slightly from that on continent.

This booklet can be a valuable companion of game managers and other professionals looking for new insights into dealing with deer problems pertaining to forestry, wildlife, and the environment.

Ryszard DZIĘCIOŁOWSKI, Department of Forest Zoology and Wildlife Management, Faculty of Forestry, Agricultural University SGGW, Rakowiecka 26/30, 02-528 Warszawa, Poland; e-mail: dzieciol@delta.sggw.waw.pl 